\title{
Non-Bacterial Thrombotic (Marantic) Endocarditis of the Aortic Valve: A Rare Cause of STEMI Revealing a Polycythemia
}

\author{
Judit Özse* (D), Rainer Knur (D) and Nicolas Von Beckerath \\ Department of Cardiology and Angiology, General Hospital Viersen, Germany
}

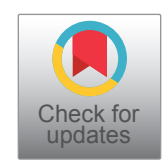

*Corresponding author: Judit Özse, Department of Cardiology and Angiology, General Hospital Viersen, 40667 Meerbusch, Dülsweg 15, Germany, Tel: +4915784504532

\section{Introduction}

Acute myocardial infarction is a major cause of death and disability worldwide. In general $4 \%$ to $7 \%$ of all patients diagnosed with AMI do not have coronary artery disease (CAD) at coronary angiography or autopsy. Embolisation as a mechanism of myocardial infarction is well known. Large diversity of aetiologies makes the precise diagnosis challenging. Its sources can be thrombi from left sided heart cavities, paradoxal embolism, tumors (myxoma, fibroelastoma), endocarditis (infective or nonbacterial thrombotic), valves (native, prosthetic) or heart catheterization or surgery [1]. Nevertheless the native aortic valve thrombosis as a source of embolisation into the coronary artery is uncommon $[2,3]$.

Here we report a case of acute myocardial infarction caused by an aortic valve thrombus that was attached to the left coronary cusp in the normal aortic valve. The thrombus was presumably caused by polycythemia.

\section{Clinical Case}

A 53-year-old man was admitted per emergency doctor for chest pain since about half on hour with radiating into the back. On the emergency ECG (Figure $1 \mathrm{a}$ and Figure $1 \mathrm{~b}$ ) was evidence of ST elevation in inferior leads. He had a hypertension and smoked till 2001. His father had "thick" blood and died on myocardial infarction. He had no cardiac history. About 10 months ago was diagnosed a polyglobulie with initial hemoglobin form $17.5 \mathrm{~g} / \mathrm{dl}$ und hematocrit from $<51 \%$, the patient was in haematological treatment. Bone marrow biopsy was done without evidence of myeloproliferative disease and myelodysplastic syn- drome, JAK-2 mutation was negative, blood level of erythropoietin was normal. His haematologist diagnosed most likely a reactive polyglobulie and he was treated symptomatic with phlebotomy. Splenectomy was done in 1984 after traffic accident.

After the diagnosis inferior STEMI he was currently treated with aspirin, heparin and P2Y12 inhibitor (prasugrel) and underwent urgent coronary angiography (Figure 2). This demonstrated a total occlusion of ramus intermedius without signs of atherosclerosis (Figure 3). Fast VT has occurred with defibrillation after insert a wire. The recanalisation of ramus intermedius was unsuccessful (Figure 4). The patient was admitted on our intensive care unit and he was treated with Ilb/ Illa inhibitor (tirofiban) by intravenous perfusion for 24 hours. Laboratory tests showed a mild polycythemia with erythrocytes $5.76 \times 10^{6} / \mu \mathrm{l}$, haemoglobin $14.9 \mathrm{~g} /$ $\mathrm{dl}$, thrombocytes $522000 / \mu \mathrm{l}$ and leucocytes $13860 / \mu \mathrm{l}$; CK elevated on a maximal level of $2343 \mathrm{U} / \mathrm{I}$, Troponin T $3264 \mathrm{pg} / \mathrm{ml}$. Transthoracic echocardiography reveals left ventricle ejection fraction $38 \%$ with lateral wall hypokinesis. Chest $\mathrm{X}$-ray with mild pulmonal vein congestion. He was treated with beta-blockers, ACE-inhibitors and eplerenon. Within search for source of embolisation, transoesophageal echocardiography was performed and showed echogenic mobile mass $(11.8 \times 5.3$ $\mathrm{mm}$ ) attached to the left coronary leaflet of aortic valve with floating in direction the left main artery (Figure 5 and Figure 6). Our differential diagnosis of the mass included fibroelastoma, fibroma, other type of tumor or thrombus. Infective vegetation was considered unlikely but not excluded. The patient didn't have fever, blood cultures were negative. The patient was embarrassed

Citation: Özse J, Knur R, Beckerath NV (2020) Non-Bacterial Thrombotic (Marantic) Endocarditis of the Aortic Valve: A Rare Cause of STEMI Revealing a Polycythemia. Int J Clin Cardiol 7:169. doi. org/10.23937/2378-2951/1410169

Accepted: March 21, 2020; Published: March 24, 2020

Copyright: (C) 2020 Özse J, et al. This is an open-access article distributed under the terms of the Creative Commons Attribution License, which permits unrestricted use, distribution, and reproduction in any medium, provided the original author and source are credited. 


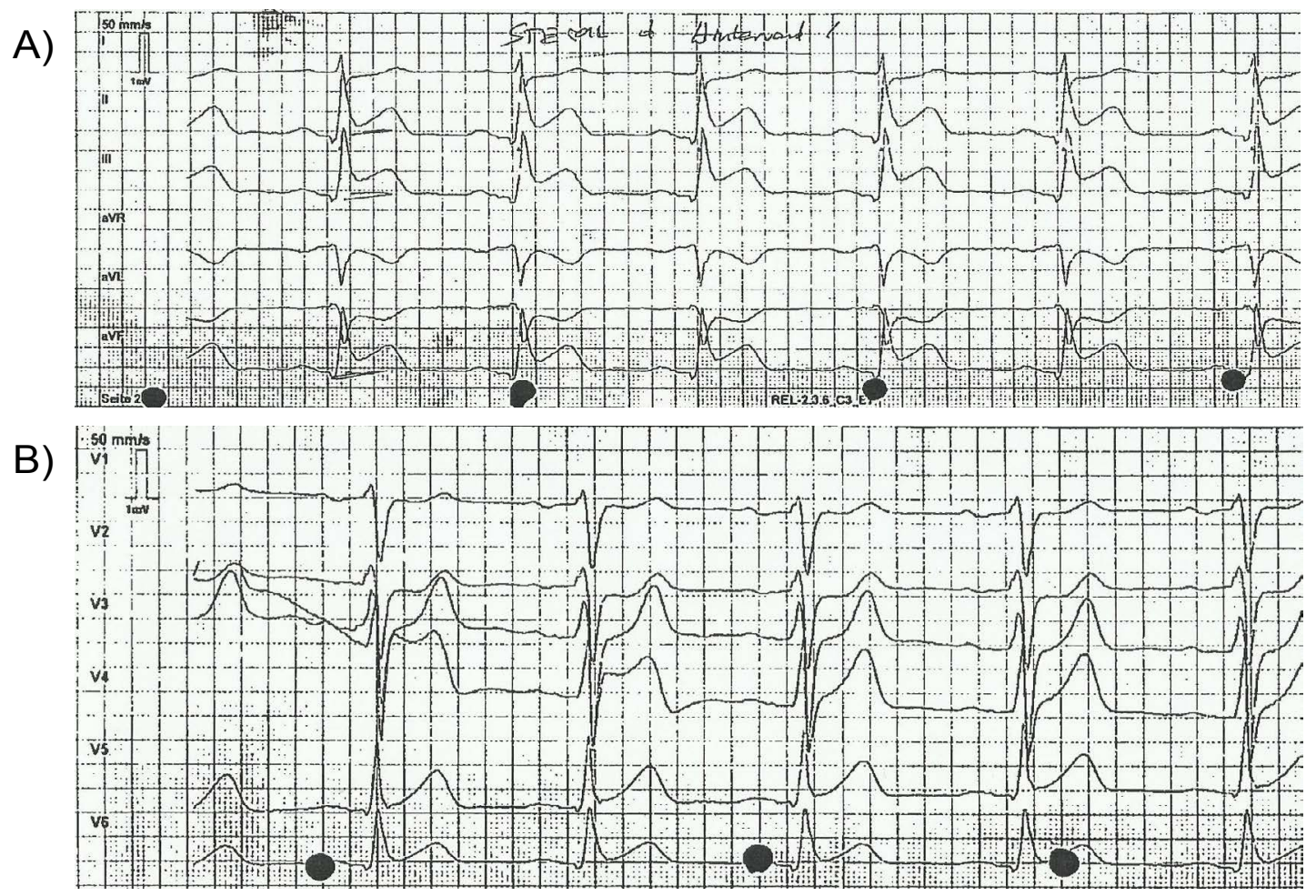

Figure 1: a,b) Emergency ECG.

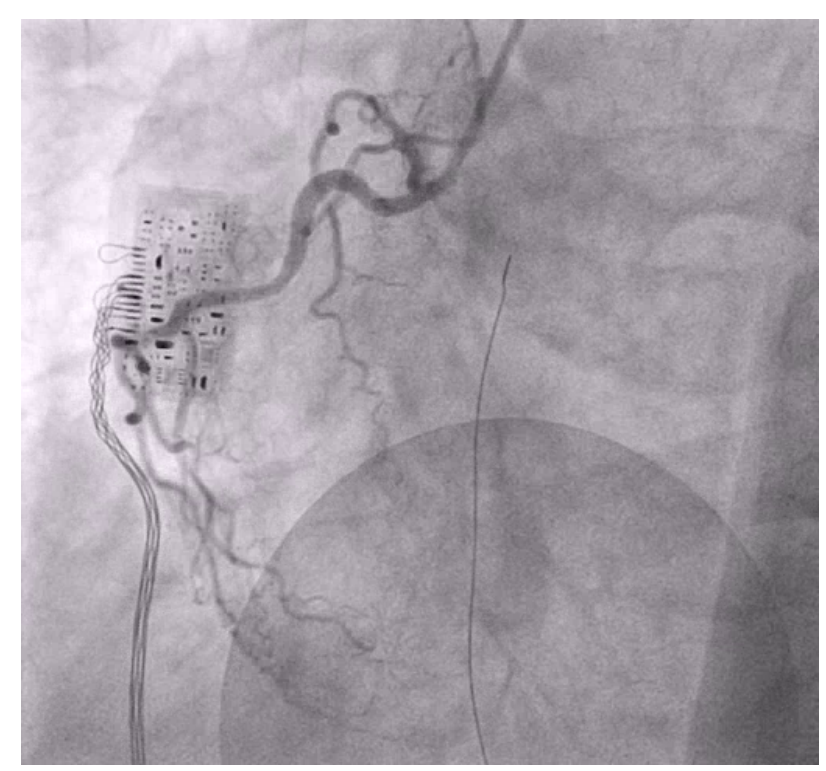

Figure 2: Right coronary artery.

into the cardiac surgery. He underwent operation under cardiopulmonary bypass and the mass of aortic valve was extirpated from the left coronary cusp of the aortic valve, it was attached with a ca. $1 \mathrm{~mm}$ thin and $5 \mathrm{~mm}$ long pedicle. Macroscopic it was a matter of a benign pediculated tumor. Histological it was fibrinous containing eosinophilic granulocytes and lymphocytes and a few plasmacytes and monocytes. The pathological analysis demonstrated a thrombus, with no evidence of neoformation or an infectious process. The postoperative period was uneventful both in vascular and cardiac items.

Laboratory test showed after the operation an ele-

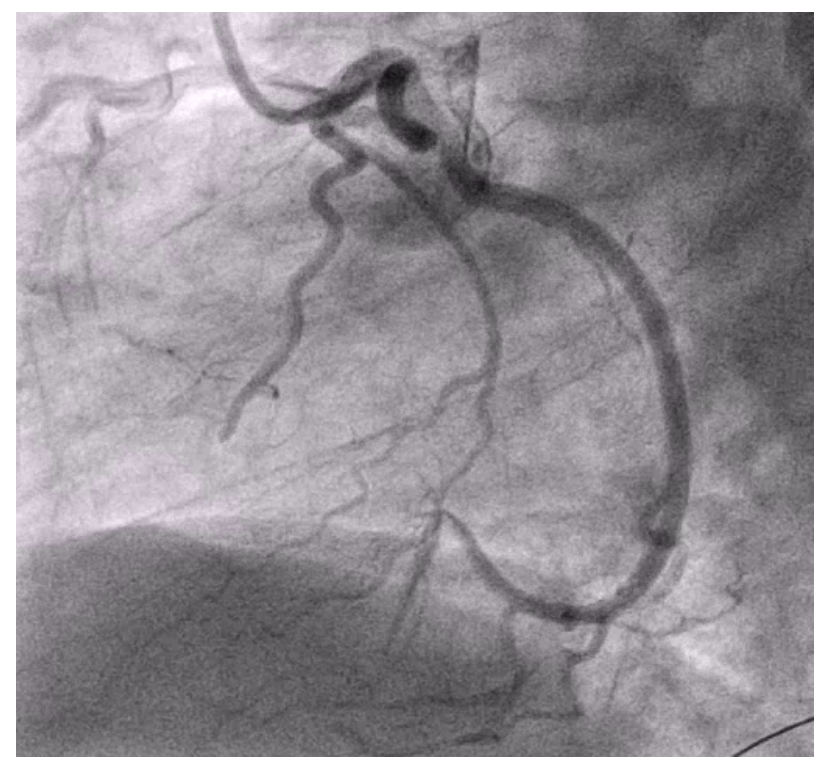

Figure 3: Left coronary artery with peripheral occlusion of ramus intermedius.

vation of the thrombocytes to $1493000 / \mu$ l. The patient was immediately treated with hydroxyurea as an antiproliferative drug and with low molecular weight heparin. Subsequently he has been taking oral anticoagulant treatment with phenprocoumon. The patient was emitted in a good clinical condition.

He is under further hematologic and cardiologic control. For further differential diagnosis of polycythemia he was in a sleep-laboratory after the cardiac rehabilitation and sleep apnoea syndrome was diagnosed and also treated. 


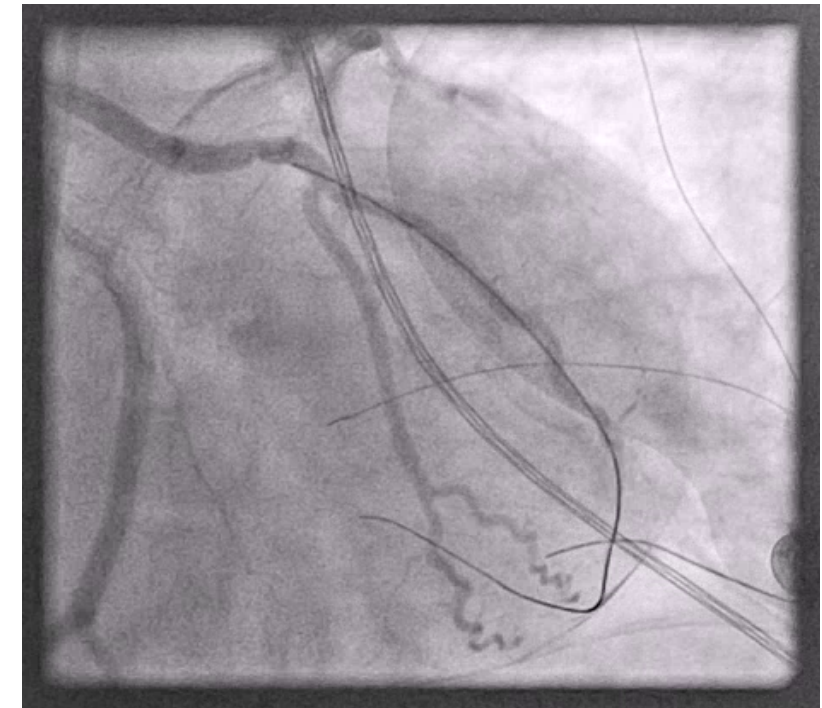

Figure 4: Left coronary artery, unsuccessful recanalisation of ramus intermedius.
He did not show further embolic events yet.

\section{Discussion}

In general $4 \%$ to $7 \%$ of all patients diagnosed with AMI do not have coronary artery disease. Coronary artery embolism should be considered as a non-atherosclerotic cause of acute myocardial infarction. Coronary artery embolism should be suspected in patients with factors or comorbidities predisposing to embolic events. Large diversity of aetiologies makes the precise diagnosis challenging. There are still no clear guidelines for diagnostic criteria such cases. Depending on aetiology, the treatment needs to be tailored individually.

There are a number of causes of coronary artery embolism. The most common cause is non-valvular atrial fibrillation. Less frequent but worth mentioning aetiologies included cardiomyopathy, valvular heart disease such as rheumatic heart disease with mitral stenosis, thrombus trough a patent foramen ovale

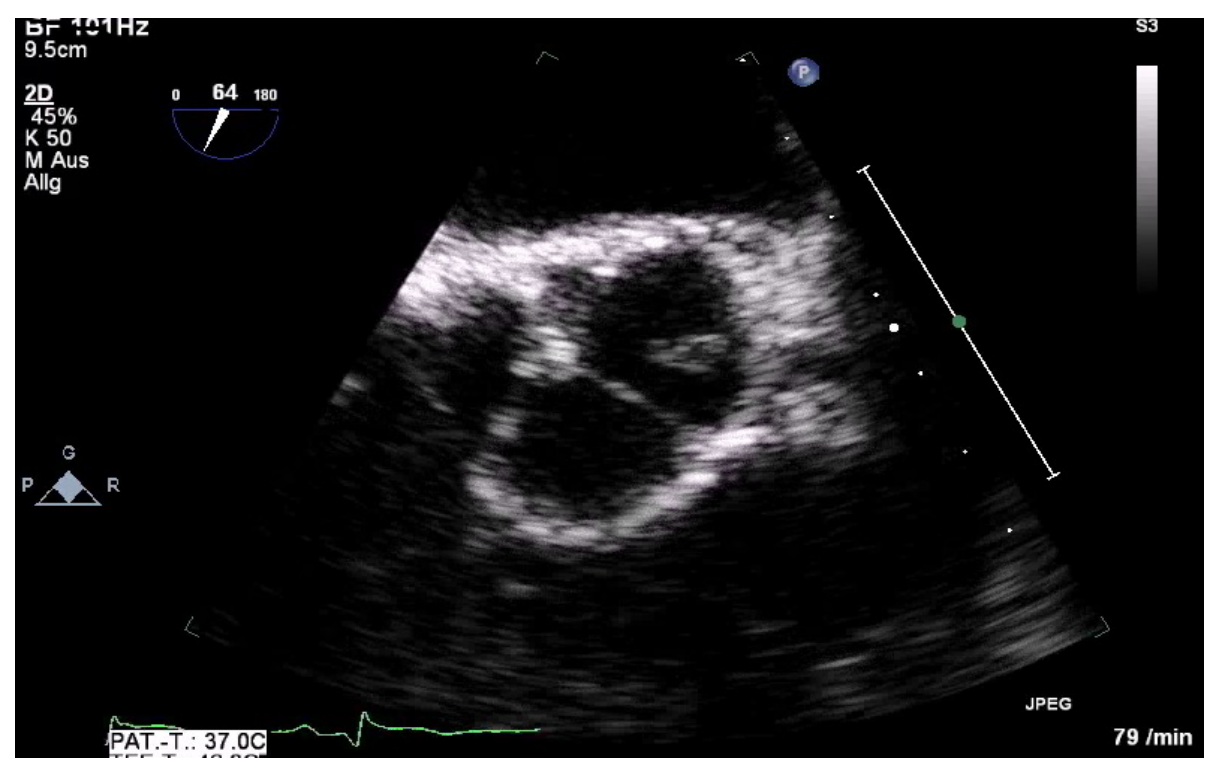

Figure 5: Transoesophageal finding of mass attached to the aortic valve with floating in direction the left main artery.

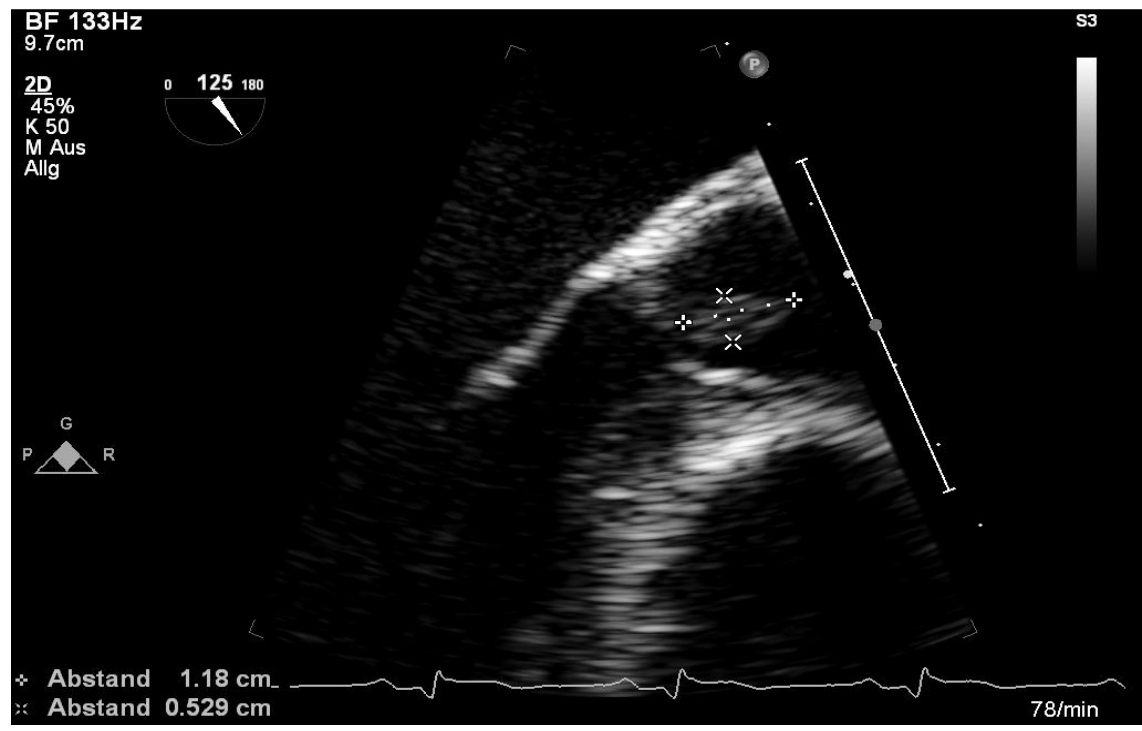

Figure 6: Transoesophageal finding of mass attached to the aortic valve. 
or atrial septal defect, infectious endocarditis, atrial myxoma, left ventricular aneurysm, iatrogenic during coronary arteriography with injection of thrombus, non-infected thromboemboli from prosthetic valve and fibromyxoma of the aortic valve [4]. A recent study by Shibata, et al. implicates cardiac embolism patients had a lower prevalence of hypertension, diabetes mellitus, dyslipidemia and smoking, and a lower number of major risk factors for cardiac artery disease than non-cardiac embolism AMI patients [5]. Recurrent thromboembolic episodes were identified in $10.4 \%$ of patients during follow up and were therefore more likely to benefit from treatment with vitamin $\mathrm{K}$ antagonists. The consequences of cardiac embolism depend both the size of the embolus and the size of the artery in which it becomes impacted. It has been long recognised that most frequently lodge in the distal parts of coronary tree leading to small but transmural myocardial infarction. In patients with presumed embolic coronary event, TEE has been recommended to identify any potential endocardial source of emboli [1]. There is no consensus about optimal treatment of patients with coronary artery embolism. The choice of the appropriate method of treatment should be individualised. Manual thrombus aspiration has been shown to be a feasible and effective strategy for the treatment of cardiac coronary embolism related infarction. Its limitation is to access the small and often tortuous vessels, where the emboli are usually located. Percutaneous transluminal coronary angioplasty (PTCA) should be considered in the case of total coronary artery occlusion. In most described cases angioplasty without stent implantation is effective enough. Oral anticoagulation should be managed due to the risk of venous origin of thrombus [6]. A thrombolytic therapy should be avoided in patients with infective endocarditis. The prognosis of coronary artery embolism depends on many factors. The 30 days cardiovascular mortality in the coronary artery embolism group is significantly lower than in patients with acute myocardial infarction due to atherosclerotic coronary artery disease. The 5-year rates of all-cause death and cardiac death in the coronary artery embolism group is unexpectedly high [5]. The reason is probably that patients without pre-existing ischaemic heart disease have poorly developed collaterals; it is commonly believed that coronary artery embolism more often leads to sudden cardiac death than coronary thrombosis in patients with atherosclerosis. Coronary embolism should be considered in all cases of acute myocardial infarction in patient without atherosclerotic coronary artery disease. As long-term outcomes indicate, coronary embolism patients represent a high-risk subpopulation of patients with acute myocardial infarction and therefore require close follow-up.

Native aortic valve thrombosis is rarely reported and usually occurs after valve injury after cardiac catheter- ization or surgery or after bacterial endocarditis [7,8]. These cases are commonly associated with anomalies, such as bicuspid valves, calcifications, stenosis or valve injury [9]. Aortic thrombosis with a normal aortic valve is extremely rare. Coagulopathy might be essential in the formation of thrombus on healthy aortic valves $[10,11]$.

Patient with polycythemia occasional have nonspecific valve thickening or marantic endocarditis. An increased hematocrit increases blood viscosity and predisposes to stasis and thrombosis. Symptoms of hyperviscosity are common when the hematocrit is greater than $60 \%$ and are indicative of low tissue flow. Polycythemia literally means too many cells in the blood (an increased hematocrit and hemoglobin) and may occur for a wide range of reasons and therefore is considered in these categories: Relative polycythemia, secondary polycythemia and primary polycythemia or polycythemia rubra vera [12]. The symptoms are very non specific like headache, weakness, pruritus, dizziness, sweating and visual disturbances. The complications of polycythemia are an increased risk of thrombosis (cerebral or peripheral) and haemorrhage [13]. A detailed history is very important in differentiating the causes of polycythemia. Primary familial and congenital polycythemia is suggested when there is history of polycythemia from childhood or many members of the family. History of chronic respiratory diseases, sleep apnoea, residing in high attitude, smoking, renal tumours, liver diseases, congenital heart diseases and EPO use are all important [14].

The management depends on the cause. Treatment consists of expansion of plasma volume (phlebotomy or erythropheresis) and chemotherapy with antiproliferative drugs such as hydroxyurea. Treatment is undertaken when the hematocrit is greater than $45 \%$ if disturbances attributable to the polycythemia are seen. Myelosuppressive drugs should be used to reduce the requirements for phlebotomy and to reduce platelet concentrations. All secondary causes should be appropriately treated [13].

\section{Conclusion}

We reported a case of a patient with acute myocardial infarction caused coronary artery embolism by a native aortic valve thrombus revealing a polycythemia.

Spontaneous native aortic valve thrombosis is infrequent. It should be taken into the consideration in the differential diagnosis of valve-associated masses und causes of systemic embolism. Transoesophageal echocardiography is of crucial importance in its detection and further management of therapy.

\section{Conflict of Interest}

No. 


\section{Statement of Equal Authors' Contribution}

Özse J conceived of the presented idea. Knur R encouraged Özse J to investigate the case and von Beckerath $\mathrm{N}$ supervised the findings of this work. All authors discussed the results and contributed to the final manuscript.

\section{References}

1. Novobílsky K, Kaucak V, Bárta J, Kryza R, Dvorackova J (2014) Native aortic valve thrombus as a source of embolisation into the coronary artery. Cor et vasa 56: e413-e416.

2. Víctor Bazán, Arturo Evangelista, Gustavo Avegliano, M Teresa González, Cristina Elorz, et al. (2002) Non-bacterial thrombotic endocarditis of the aortic valve in a young woman. Rev Esp Cardiol 55: 1333-1336.

3. Shi-Min Yuan, Hua Jing, Jacob Lavee (2009) Tumors and tumor-like lesions of the heart valves. Rare Tumors 1: e35.

4. Minkwan Kim, Suk-Hyun Kim, Sang Yi Moon, Eu Gene Jeong, Eui Han Jung, et al. (2014) Native aortic valve thrombosis resembling papillary fibroelastoma. J Cardiovasc Ultrasound 22: 148-150.

5. Shibata T, Kawakami S, Noguchi T, Tanaka T, Asaumi Y, et al. (2015) Prevalence, clinical features, and prognosis of acute myocardial infarction attributable to coronary artery embolism. Circulation 132: 241-250.

6. Laurent Barandon, Philippe Clerc, Christophe Chauvel, Philippe Plagnol (2004) Native aortic valve thrombosis: A rare cause of acute ischemia of the lower limb. Interact Cardiovasc Thorac Surg 3: 675-677.

7. Yamawaki M, Shimoyama M, Furuse $Y$, Kinugasa $Y$, Ogino $\mathrm{K}$, et al. (2007) Floating thrombus arising from left sinus of Valsalva induced intermittent occlusion of left coronary artery and caused cardiogenic shock. Int J Cardiol 114: 272-273.

8. Michael H Crawford, John P DiMarco, Walter J Paulus (2009) Cardiology. ( ${ }^{\text {rd }}$ edn), Elsevier, ISBN: 978-0-72343485-6.

9. Massetti M, Babatasi G, Saloux E, Bhoyroo S, Grollier G, et al. (1999) Spontaneous native aortic valve thrombosis. J Heart Valve Dis 8: 157-159.

10. Elisabetta Grolla, Michele Dalla Vestra, Luca Bonanni, Ada Cutolo, Fausto Rigo (2015) A rare case of aortic valve thrombosis in patient with idiopathic hypereosinophilic syndrome. Case Rep Cardiol 2015: 607107.

11. Jobic $\mathrm{Y}$, Provost K, Larlet JM, Mondine P, Gilard M, et al. (1999) Intermittent left coronary occlusion caused by native aortic valve thrombosis in a patient with protein $\mathrm{S}$ deficiency. J Am Soc Echocardiogr 12: 1114-1116.

12. Swerdlow SH, Campo E, Harris NL, Jaffe ES, Pileri SA, et al. (2008) WHO classification of tumours of haematopoietic and lymphoid tissues. IARC, Lyon, France.

13. Mathew Thomas, K Pavithran (2010) A clinical approach to polycythemia. Medicine.

14. McMullin MF (2008) The classification and diagnosis of erythrocytosis. Int J Lab Hematol 30: 447-459. 